\title{
Closing the loop between mitigation, impacts and adaptation
}

\section{An editorial essay}

\author{
Martin Parry
}

Received: 22 May 2009 / Accepted: 14 July 2009 / Published online: 15 August 2009

(C) Springer Science + Business Media B.V. 2009

In its Fourth Assessment (2007) the IPCC was unable to address successfully the single most important policy question about confronting climate change: "What combinations of emissions reduction and adaptation can best reduce the impacts of climate change?" It is a hugely important question because it requires knowledge that is needed to underpin the agreement being sought at the UNFCCC summit this December (2009) in Copenhagen. Its omission stems from two things: (a) failure to frame the question that would help synthesise current knowledge, and (b) lack of knowledge itself on the connections between mitigation, impacts and adaptation. The first failing is easier to remedy than the second.

\section{Framing questions about the loop}

The key question needs decomposing into three parts for each IPCC Working Group (WG). These sub-questions are: What is the range of different emissions reductions that might be achieved at varying cost? (a key sub-question for WG III), What would be the climate outcomes of these different mitigative actions? (WG I), and: What would be the impacts not avoided by mitigation and thus needing either to be avoided by adaptation or to be borne? (WG II). Only WGIII addressed its key sub-question successfully in the IPCC Fourth Assessment.

There is a straightforward solution. The draft chapter outlines of each IPCC Working Group were examined and then approved by WG sub-sections of plenary sitting, not together, but in parallel. The separately-approved WG outlines were then accepted by the full Plenary with little discussion, and certainly not with the scrutiny needed to see how they would knit together. In the next (fifth) IPCC assessment, this could easily be remedied by ensuring close scrutiny by Plenary of the three WG outlines together.

M. Parry $(\varangle)$

Grantham Institute, Imperial College London, London, UK

e-mail: martin@mlparry.com 


\section{Building knowledge about the loop}

Knowing more about 'the loop' is a greater challenge. The origin of the problem stems, I believe, from scientists not having a common conception of it. We know that the three components (impacts, mitigation, adaptation) are inter-connected, and we can picture how they are part of a mix of actions and outcomes (see, for example, Fig. 1). But sometimes the actions can be complementary and at others they can trade off each other; and it is not at all clear what mix of different amounts of mitigation and adaptation would best meet given targets of impact avoidance. Indeed, many scientists would not accept that any such optimal mix exists.

In the absence of sufficient knowledge, we tend to assume that the wisest course of action involves: (a) reducing emissions as much as we can afford so as to keep impacts and adaptation costs to the minimum over the long term, (b) adapting to most of the remaining impacts so as to minimise damage to society and the environment, and (c) bearing the costs of the unavoidable residual damage (which includes impacts we cannot adapt to or we judge not worth adapting to). This explains, in the schematic of Fig. 1, why the nexus of actions is located to the right of the figure, in the touching belief that most impacts will be avoided. In fact even this remains in doubt, some analysts being of the view that about two-thirds of the cost of climate change will need to be borne rather than avoided by adaptation (Parry et al. 2009).

\section{Research on closing the loop}

Since the 2007 IPCC Assessment, partly in response to gaps in the Assessment and also driven by the need to answer urgent questions from policymakers, a number of new analyses have been completed of the climate outcomes for varying strategies of emissions reductions (e.g., Hansen et al. 2008; Allen et al. 2009; Meinshausen et al. 2009; Parry et al. 2009; Schneider 2009). When we super-impose these climate out-

Fig. 1 Schematic on inter-connection between climate change impacts, mitigation and adaptation (source: author)

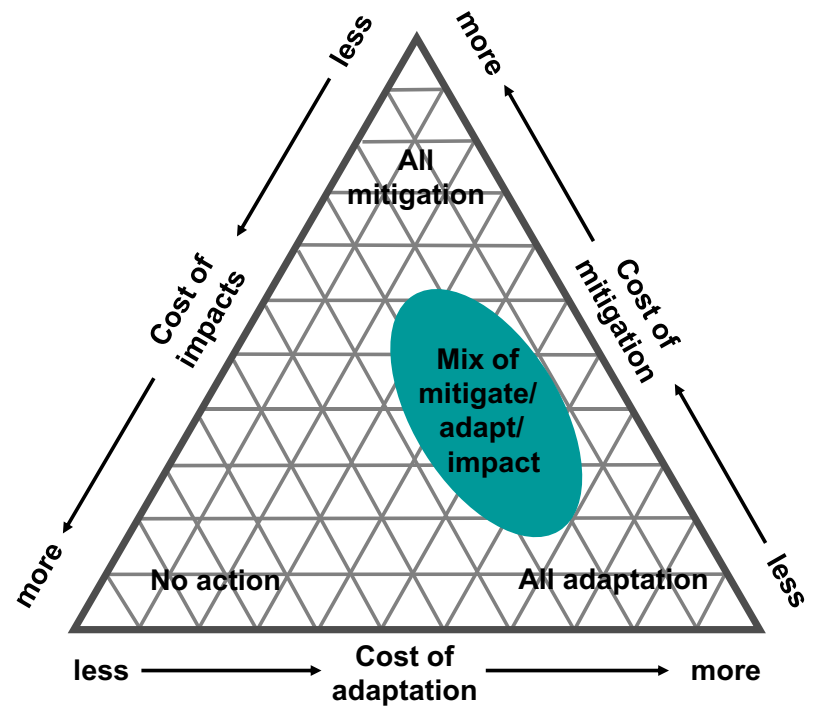


comes on the table of impacts summarised in the 2007 Working Group II assessment of the IPCC (IPCC 2007, Technical Summary), we can explore the impacts avoided or not by mitigation, as well as the amounts of adaptation needed to keep residual impacts to a minimum.

What follows is an example of closing the loop, where we examine the effect of different start dates of emissions reduction in avoiding impacts from climate change. Figure 2 shows the projected global temperature increases using a simple Earthsystem model (Lowe et al. 2009). Here we assume rates of global emissions, which are currently increasing at c. $3 \%$ per year, are transformed to a $3 \%$ annual reduction. The emissions peak or downturn is at varying dates, one resulting from immediate action with an emissions downturn in 2015 (and global mean temperature projected to peak around 2065); one for delayed action with an emissions downturn in 2025, and a temperature peak around 2080; and one for a further delay in action with a 2035 downturn and peak temperatures around 2100 (Parry et al. 2009). If the annual 3\% pa emission reduction is maintained over two centuries, which would be an immense task, then global temperatures could recover to $1.5^{\circ} \mathrm{C}$ by 2200 and $1.0^{\circ} \mathrm{C}$ by 2300 , a state advocated by James Hansen and others (Hansen et al. 2008).

Figure 3, which plots these climate projections onto impacts is, in effect, a sliding scale of impacts mapped against mitigation. It can be used to describe the impact outcomes of different strategies, including different amounts of emissions reductions as well as different timings of action. The vertical lines represent projected median temperature outcomes, so that impacts to the right of the lines are as likely as not to be avoided by mitigation, and vice versa for impacts to the left. The area to the left is thus the 'adaptation field', the area of potential impacts which either must be borne or adapted to.

From this, we can see that, assuming the strongest possible action that can be expected from Copenhagen in December 2009 is one which leads to a global emissions downturn in 2015 and a 3\% annual reduction continuing thereafter, there

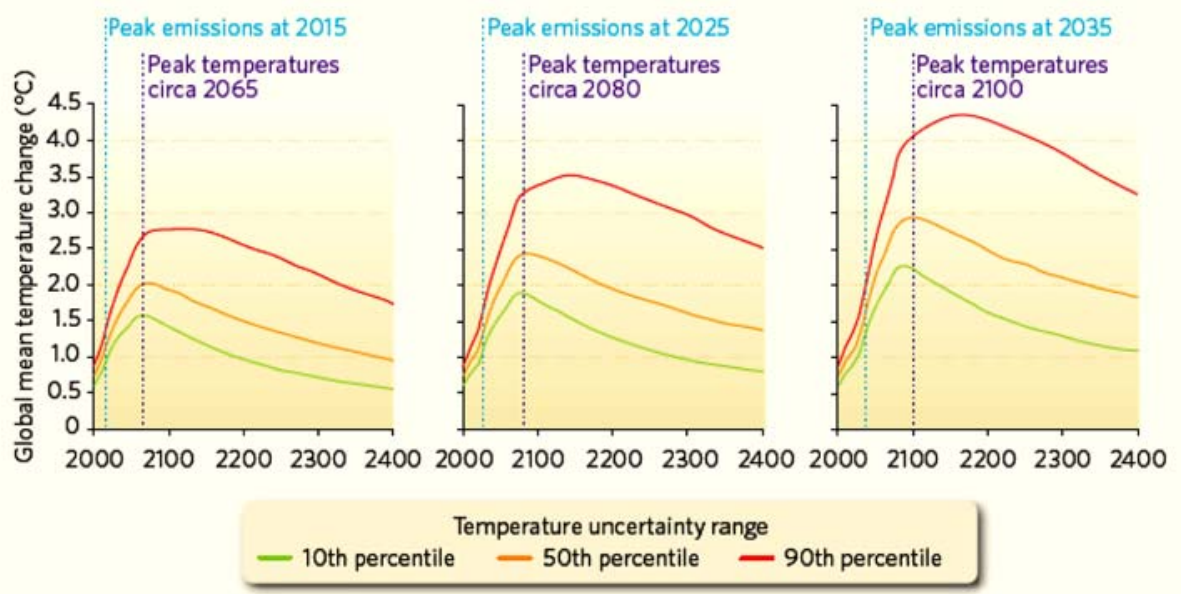

Fig. 2 Global average surface temperature scenarios of peak emissions at different dates $(2015,2025$ and 2035) with 3\%-per-year reductions in greenhouse gas emissions [source: Parry et al. 2009] 


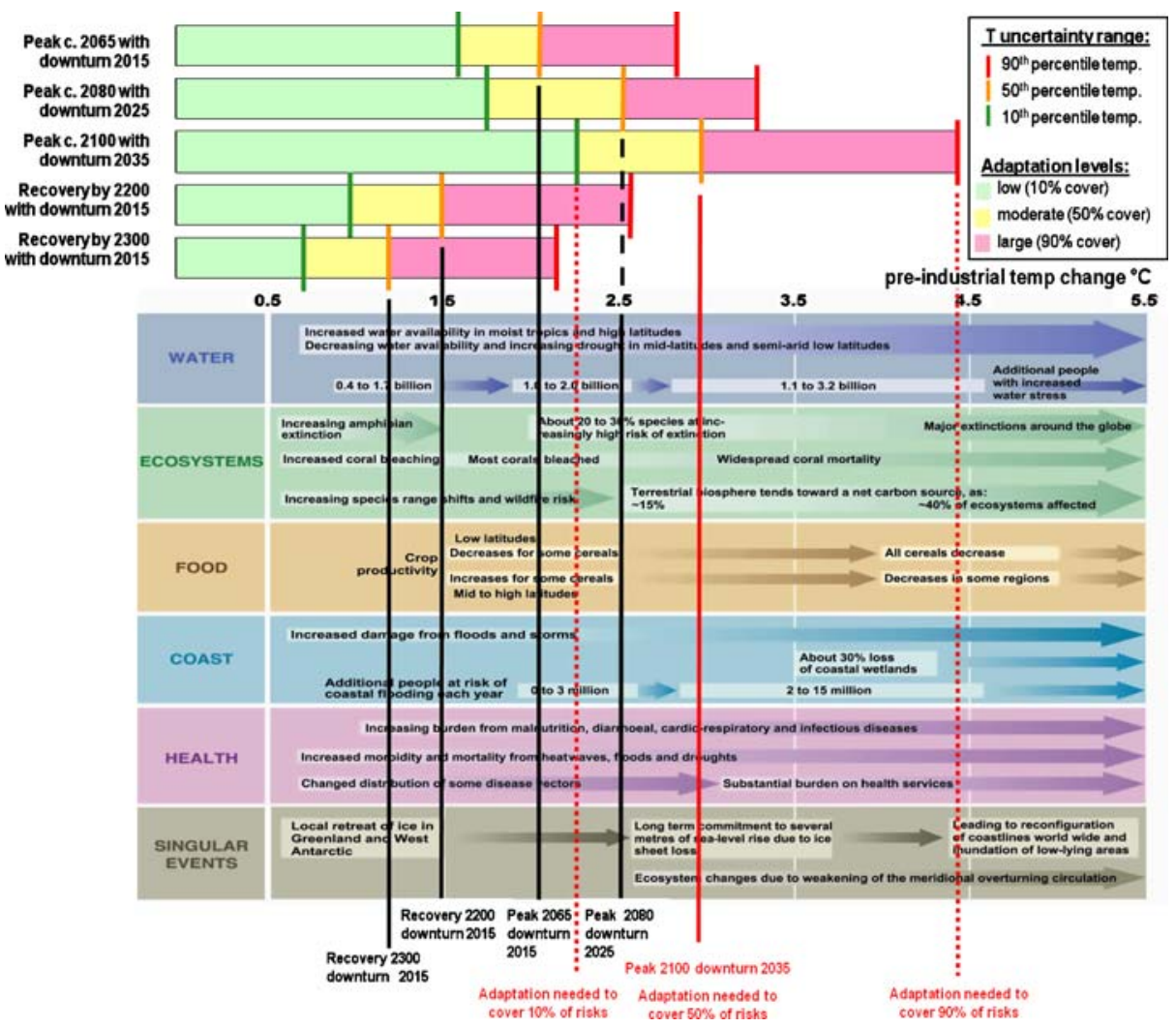

Fig. 3 Selected global impacts from warming associated with $3 \%$ p.a. global emissions reduction, and with global emissions downturns in 2015, 2025 and 2035. Black vertical lines show (a) median projected global temperatures at their peak for different emissions downturn times, and (b) median temperatures at recovery times in 2200 and 2300. Red vertical lines show different adaptation needs for emissions downturn in 2035 and peak temperature c. 2100 . Horizontal bars indicate uncertainty range for temperature, and adaptation needs for 10,50 and $90 \%$ coverage of expected climate risk. (source: Author, with impacts table from IPCC WGII Technical Summary, 2007)

is approximately an even chance of exceeding the G8 and EU target of $2^{\circ} \mathrm{C}$. The potential impacts, even under this scenario of strong mitigative action, are substantial; for example, 1 to 2 billion people become short of water. The consequences for delayed or reduced action can also be inferred from Fig. 3.

\section{Bringing adaptation into the loop}

There is a substantial range of uncertainty surrounding the temperature outcomes for different courses of mitigative action, shown by horizontal bars in Fig. 3, and these represent a major challenge for adaptation. Since adaptation costs increase steeply, sometimes even quadratically, with climate change there are difficult decisions to be made about the extent of cover to prepare for. 
In Fig. 3 we assume that high levels of adaptation are needed to cover $90 \%$ of impacts, moderate levels of adaptation would cover $50 \%$, and low levels would cover only $10 \%$ of impacts. On this basis, for example, if global emissions did not peak until 2035 and if we wished to cover $90 \%$ of expected impacts, then we should be planning to adapt to at least $4^{\circ} \mathrm{C}$ of warming.

It is possible, then, by scaling impacts against increases in temperature and by inferring the risk of impact from the uncertainty of climate outcomes, to begin to close the loop of mitigation, impacts and adaptation. But we have barely made a start, and it is urgent that new research is focussed on this problem. The reason is that this question, which I started with, is at the heart of the policy challenge: "What combinations of emissions reduction and adaptation can best reduce the impacts of climate change?"

\section{References}

Allen M et al (2009) Warming caused by cumulative carbon emission towards the trillionth tonne. Nature 458(7242):1163-1166

Hansen J et al (2008) Target atmospheric $\mathrm{CO}_{2}$ : where should humanity aim? J Atmos Sci 2:217-223 Lowe JA et al (2009) How difficult is it to recover from dangerous levels of global warming? Environ Res Lett 4:014012. doi:10.1088/1748-9326/4/1/014012

Meinshausen et al (2009) Greenhouse-gas emission targets for limiting global warming to 2 deg C. Nature 458(7242):1158-1162

Parry ML, Lowe JA, Hanson CH (2009) Overshoot, adapt and recover. Nature 458(7242):1102-1103

Schneider SH (2009) The worst-case scenario. Nature 458(7242):1104-1105 